\title{
Transformasyon Grafların Komşu İzole Saçılım Sayısı
}

\author{
Ersin ASLAN*1®, Büşra AÇAN² \\ ${ }^{1}$ Manisa Celal Bayar Üniversitesi, Hasan Ferdi Turgutlu Teknoloji Fakültesi, Yazılım Mühendisliği, 45400, Manisa, \\ Türkiye \\ ${ }^{2}$ Manisa Celal Bayar Üniversitesi, Fen Bilimleri Enstitüsü, 45400, Manisa, Türkiye
}

(Alınıș / Received: 16.05.2019, Kabul / Accepted: 08.07.2019, Online Yayınlanma / Published Online: 30.08.2019)

Anahtar Kelimeler

Graf teori,

Zedelenebilirlik,

Bağlantı sayısı,

Saçılım sayısı
Özet: Bir ağın (iletişim, taşıma, internet, vb.) merkezleri ya da bağlantı hatları bazı durumlarda zarara uğrayabilir. Bu durumlar, ağda bazı sorunlar ortaya çıkmasına sebep olabilir. Burada en çok merak edilen soru ise; bir ağda iletişim durana kadar ağın ne kadar sürede nasıl dayanacağıdır. Zedelenebilirlik, iletișim ağında belli merkezlerin ya da bağlantıların zarar görmesinden sonra, iletişim kesilene kadar geçen süredeki ağın dayanma gücünün hesaplanmasına zedelenebilirlik değeri denir. Bu çalıșmada komşu izole sayısı incelenmiştir. Ardından bazı grafların transformasyon hallerinin komşu izole saçılım sayıları hesaplanmıștır.

\section{Neighbor Isolated Scattering Number of Transformation Graphs}

\section{Keywords}

Graph theory,

Vulnerability,

Connectivity,

Scattering number

\begin{abstract}
A network (communication, mobile, internet etc.) centers or connection lines may be damaged in some cases may come in. These situations can cause some extreme in the network to appear. Most curious here the question is how long the network will last until communication is stopped. The vulnerability of a graph is a determination that includes certain properties of the graph not to be damaged after the removal of a number of vertices. In this article, we consider the neighbor isolated scattering number of transformation graphs.
\end{abstract}

\section{Giriş}

Graf teori, modern hayatın karmaşık ve geniş kapsamlı birçok problemin çözümü için kullanılmaktadır. Graf, dügümler ve bu dügümleri birbirine bağlayan kenarlardan oluşan bir tür ağ yapısıdır. Bir graf, düğümlerden (köşeler) ve bu düğümleri birbirine bağlayan ayritlardan (kenarlardan) oluşur. Öncelikle graf teoride kullanılan bazı temel tanımları verelim. Bu tanımlar Harary [1] kitabından alınmıştır. Herhangi bir $G$ grafının bir $v$ tepesine bağlı ayrıtların sayısına, o tepenin derecesi denir ve $\operatorname{deg}(v)$ ile gösterilir. Bir $G$ grafının bir $v$ tepesine bağlı ayrıtların sayısı sıfır ise o tepeye izole tepe denir. G grafının herhangi bir u tepesinin açık komşuluğu, $N(u)=\{v \in V(G) \mid v \neq$ $u$, u ve v komşu $\}$ ve kapalı komşuluğu, $N[u]=\{u\} \cup$ $N(u)$ şeklinde tanımlanır. $N[u]$ kapalı komşuluğu graftan atılan $u$ tepesine karşılık gelen tanım subverted tepedir. Tüm tepeleri subverted olan $X=$ $\left\{u_{1}, u_{2}, \ldots, u_{m}\right\}$ kümesine, $\mathrm{G}$ grafının subversion stratejisi denir. X kümesindeki tüm tepeler, graftan subverted edildiğinde geriye kalan graf, bağlantısız bir graf, bir klik ya da boş graf olabilir. $G_{1}$ ve $G_{2}$ iki graf olsun. İki grafın birleşimi (union) $G=G_{1} \cup G_{2}$, tepeler kümesi $\mathrm{V}(\mathrm{G})=\mathrm{V}\left(\mathrm{G}_{1}\right) \cup \mathrm{V}\left(\mathrm{G}_{2}\right)$ ve ayritlar kümesi $\mathrm{E}(\mathrm{G})=\mathrm{E}\left(\mathrm{G}_{1}\right) \cup \mathrm{E}\left(\mathrm{G}_{2}\right)$ dir. $G$ bir graf ve $G$ nin tepeler kümesi $V(G)$ olsun. $\mathrm{V}(\mathrm{G}), \mathrm{G}$ grafının tepeler kümesi olmak üzere, $V(G)$ kümesinde birbirine komşu olmayan maksimum tepe sayısına bağımsızlık sayısı denir ve $\alpha(G)$ ile gösterilir.

Graf teori uygulamaları modern hayatın karmaşık ve geniş kapsamlı birçok problemin çözümü için kullanılmaktadır. Bu uygulamalar; ekonomi, yönetim bilimi, satış pazarlama, bilgi iletimi, taşıma planlaması gibi alanları kapsamaktadır. Ayrıca kimya, elektrik mühendisliği, mimarlık gibi sayısal alanlarda da uygulamaları vardır. Graf teorisi problemleri tanımlama ve yapısal olarak ilişkileri belirlemekte de faydalıdır. Graf teori için önemli konulardan biri de zedelenebilirlik kavramıdır. Bir iletişim ağında belli merkezlerin ya da bağlantıların zarar görmesinden sonra, iletişim kesilene kadar geçen süredeki ağın dayanma gücünün hesaplanmasına zedelenebilirlik değeri denir. Bir ağdaki merkezlerin zarar görmesi sonucunda, bu merkezlerin komşularının da etkilendiği bir ağ casus ağı (spy network) olarak adlandırılır. Çünkü bir casus ağ ele geçirildiğinde, bu casus ağ ile etkileşimde olan kişilerin de güvenliği 
artık tehlikededir. Bu nedenle var olan graf bu ağl temsil ettiğinden, sadece tepeler yerine, tepelerle birlikte bu tepelere komşu olan tepelerde graftan atılır. Zedelenebilirlik değerlerini incelemek için bazı ölçümler tanımlanmıştır, bazıları komşu bağlantı sayısı [2], komşu saçılım sayısı [3], komşu izole saçılım sayısıdır [4]. Grafların zedelenebilirliği ile ilgili [5-14] makaleleri gibi birçok çalışmalar yapilmiştır.

Bazı zedelenebilirlik ölçümlerinin tanımlarını verelim.

Tanım 1.1. $X$, bir $G$ grafinın tepelerinin subversion stratejisi olmak üzere $G$ grafını bağlantısız hale getirmek için atılması gereken en az tepe sayısına komşu bağlantılılık sayısı (neighbor connectivity) denir ve $\operatorname{VNC}(G)$ ile gösterilir ayrıca

$$
\operatorname{VNC}(G)=\min _{X \subseteq \mathrm{V}(\mathrm{G})}\{|\mathrm{X}|\}
$$

olarak tanımlanabilir [2].

Tanım 1.2. $\mathrm{X}$, bir $\mathrm{G}$ grafının subversion stratejisi ve $w(G / S), G / S$ grafının bileşen sayısı olmak üzere $\mathrm{G}$ grafının komşu saçılım sayısı (neighbor scattering number)

$$
\operatorname{VNS}(G)=\max _{X \subseteq \mathrm{V}(\mathrm{G})}\{w(G / \mathrm{X})-|\mathrm{X}|\}
$$

olarak tanımlanır [3].

Tanım 1.3. $\mathrm{X}$, bir $\mathrm{G}$ grafının subversion stratejisi ve $\mathrm{m}(G / S), G / S$ grafının en büyük boyutlu bileșenin tepe sayısı olmak üzere G grafının komşu bütünlük değeri (neighbor integrity number)

$$
\operatorname{VNI}(G)=\max _{X \subseteq \mathrm{V}(\mathrm{G})}\{|\mathrm{X}|+\mathrm{m}(G / \mathrm{X})\}
$$

olarak tanımlanır [5].

$\mathrm{Bu}$ çalışmada öncelikle transformasyon grafının tanımı verilmiştir. 3. bölümde komşu izole saçılım sayısının tanımı ve bazı genel teoremler verilmiştir. 4 . bölümde $P_{n}^{+++}, C_{n}^{+++}, \quad K_{1, n}^{+++}, P_{n}^{-++}, C_{n}^{-++}$ transformasyon graflarının komşu izole saçılım sayıları incelenmiştir.

\section{Transformasyon Graflar}

$\mathrm{Bu}$ bölümde öncelikle $G^{x y z}$ transformasyon grafının tanımı verilmiştir. Daha sonra var olan $G$ grafının örnekleri olarak $P_{n}$ ve $C_{n}$ graflarının transformasyon hali olan $P_{n}^{+++}, P_{n}^{-++}, K_{1, n}^{+++}, K_{1, n}^{-++}, C_{n}^{+++}, C_{n}^{-++}$ graflarınının komşu izole saçlım sayıları hesaplanmiştır.

Tanım 2.1. [6] $G=(V(G), E(G))$ bir graf ve $x, y, z$ değerleri + veya - değerlerini alabilen üç değişken olsun. $G^{x y z}$ transformasyon grafının tepeler kümesi
$V(G) \cup E(G)$ olan ve $a, b \in V(G) \cup E(G)$ olmak üzere $k$ ve $l \operatorname{nın} G^{x y z}$ de komşu olması için gerek ve yeter koşul:

1. $a, b \in V(G)$ olmak üzere $a$ ve $b, x=+$ ise $G$ de komşudur; $k$ vel; $x=-$ ise $G$ de komşu değildir.

2. $a, b \in E(G)$ olmak üzere $a$ ve $b, y=+$ ise $G$ de komşudur; $k$ ve $l$; $y=-$ ise $G$ de komşu değildir.

3. $a \in V(G), b \in E(G)$ olmak üzere $a$ ve $b, z=+$ ise $G$ de iliş̧kilidir; $k$ ve $l ; z=-$ ise $G$ de ilişkili değildir.

Şekil 1 'de $C_{5}$ grafının transformasyon hali olan $C_{5}^{-++}$ grafi verilsin.
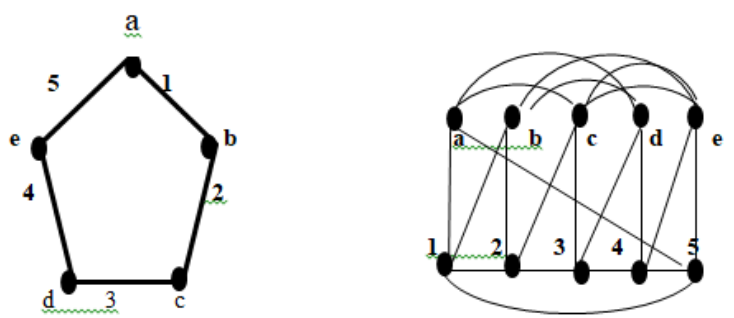

Şekil 1. $C_{5}$ grafının transformasyonu $C_{5}^{-++}$

\section{Komşu İzole Saçılım Sayısı}

Bir grafda komşu izole saçılım sayısı; grafta bazı merkezler zarar gördüğünde, bu merkezlerin kapalı komşuluğunun da zarar gördüğünü kabul ederek, geriye kalan graftaki izole tepelerin sayısı ile graftan atılan tepe sayısının farkının maksimum değerinin alınmasına dayanır.

Tanım 3.1. $G$ bir graf ve $G$ nin herhangi bir alt strateji kümesi $\mathrm{X}$ olsun. $\mathrm{i}(\mathrm{G} / \mathrm{X}), \mathrm{G} / \mathrm{X}$ grafindaki kalan izole bileşen sayısı olmak üzere $G$ grafının komşu izole saçılım sayısı

$$
\mathrm{NIS}(\mathrm{G})=\max \{\mathrm{i}(\mathrm{G} / \mathrm{X})-|\mathrm{X}|: \mathrm{i}(\mathrm{G} / \mathrm{X}) \geq 1\}
$$

olarak tanımlanır [4].

Aşağıda bilinen bazı izole saçılım sayısı ile ilgili teoremler verilmiştir.

Teorem 3.1. [4] G grafı n tepeli bağlantılı bir graf ise

$$
N I S(G) \geq 2-n
$$

Teorem 3.2. [4] $G$ grafı n tepeli bağlantılı bir graf ise

$$
N I S(G) \leq n-2 V N C(G)
$$

Teorem 3.3. [4] $G$ grafı n tepeli bağlantılı bir graf ve $\delta(G), G$ grafının minimum derece sayısı ise;

$$
N I S(G) \leq n-\operatorname{VNC}(G)(\delta(G)+2)
$$

Teorem 3.4. [4] $G$ grafı bağlantılı graf ve $\alpha(G)$ ise $G$ grafının bağımsızlık sayısı olsun. Buradan 


$$
N I S(G) \leq \alpha(G)-\operatorname{VNC}(G)
$$

Teorem 3.5. [4] $G$ bağlantılı grafı ve $G$ grafının minimum dereceli tepesi ise

$$
N I S(G) \geq 1-\delta(G)
$$

Teorem 3.6. [4] Bağlantılı $G$ grafı verilsin. Daha sonra

$$
N I S(G) \leq V N S(G)
$$

Teorem 3.7. [4] Herhangi bir $G$ grafı için,

$$
N I S(G) \geq 1-\operatorname{VNI}(G)
$$

\section{Transformasyon Grafların Komşu İzole Saçılım Sayısı}

$\mathrm{Bu}$ bölümde, $P_{n}^{+++}, P_{n}^{-++}, K_{1, n}^{+++}, K_{1, n}^{-++}, C_{n}^{+++}, C_{n}^{-++}$ grafları transformasyon graflar olmak üzere bu grafların komşu izole dağılım sayıları incelenecektir.

Teorem 4.1. $P_{n}^{+++}, P_{n}$ yol grafinın transformasyon grafı ve $n \geq 5$ olmak üzere

$$
\operatorname{NIS}\left(P_{n}^{+++}\right)=\left\{\begin{array}{c}
1, n \equiv 1(\bmod 3) \\
0, n \equiv 0,2(\bmod 3)
\end{array}\right.
$$

İspat. $P_{n}^{+++}$transformasyon grafi, $V(G) \cup$ $E(G)$ tepeler ve ayritlar kümesine sahiptir. $\mathrm{X} \subseteq$ $V\left(P_{n}^{+++}\right), P_{n}^{+++}$, grafının bir subversion stratejisi ve $|X|=r$ graftan atılan tepe sayısı olsun. İspat $n$ tepe sayısına bağlı olarak iki durumda incelenmelidir.

Durum 1. $n \equiv 1(\bmod 3)$ olsun. $|X|=\mathrm{r}$ tepe seçilirse geriye kalan tepe sayısı

$$
i\left(P_{n}^{+++} / X\right) \leq r+1
$$

olur. Buradan

$$
i\left(P_{n}^{+++} / X\right)-|X| \leq r+1-r=1
$$

elde edilir. Kolayca görülür ki $r=\frac{n-1}{3}$ seçildiğinde

$$
i\left(P_{n}^{+++} / X\right)=\frac{n+2}{3}
$$

olur. Tanımdan;

$$
\operatorname{NIS}\left(P_{n}^{+++}\right)=\frac{n+2}{3}-\frac{n-1}{3}=1
$$

elde edilir.

Durum 2. $n \equiv 0,2(\bmod 3)$ olsun. $|X|=\mathrm{r}$ tepe seçilirse izole tepe sayısı en fazla $r$ tanedir.

$$
i\left(P_{n}^{+++} / X\right)-|X| \leq r-r=0
$$

elde edilir.

- $n \equiv 0(\bmod 3)$ olduğunda ;

$r=\frac{n}{3}$ tane tepe graftan atılırsa $i\left(P_{n}^{+++} / X\right)=\frac{n}{3}$ olur. Tanım gereği

$$
\operatorname{NIS}\left(P_{n}^{+++}\right)=\frac{n}{3}-\frac{n}{3}=0
$$

olarak bulunur.

- $n \equiv 2(\bmod 3)$ olduğunda;

$r=\frac{n-2}{3}$ tane tepe graftan atılırsa $i\left(P_{n}^{+++} / X\right)=\frac{n-2}{3}$ olur. Buradan

$$
N I S\left(P_{n}^{+++}\right)=\frac{n-2}{3}-\frac{n-2}{3}=0
$$

elde edilir.

Teorem 4.2. $K_{1, n}^{+++}, n+1$ tepeli $K_{1, n}$ yıldız grafının transformasyon grafı olmak üzere

$$
\operatorname{NIS}\left(K_{1, n}^{+++}\right)=n-2 \text {. }
$$

İspat. $K_{1, n}^{+++}$yıldız grafın transformasyon grafı olmak üzere; $\mathrm{X}, K_{1, n}^{+++}$grafının kapalı komşuluğunun subversion stratejisi olsun. $|X|=r$ şeklinde tepe seçilirse $i\left(K_{1, n}^{+++}\right) \leq n-1$ olur. Buradan

$$
i\left(K_{1, n}^{+++} / X\right)-|X| \leq n-1-r
$$

elde edilir $\mathrm{f}(\mathrm{r})=n-1-r$ fonksiyonu azalan fonksiyon olup maksimum değerini $r=1$ değerinde alır. Buradan

$$
\operatorname{NIS}\left(K_{1, n}^{+++}\right) \leq n-2
$$

olur.

Burada $|\mathrm{X}|=1$ olacak şekilde öyle bir X kümesi vardır buaradan $i\left(K_{1, n}^{+++}\right)=n-1$ olup

$$
\operatorname{NIS}\left(K_{1, n}^{+++}\right)=n-2
$$

elde edilir.

Teorem 4.3. $C_{n}^{+++}, C_{n}$ çevre grafinın transformasyon grafı olmak üzere;

$$
\operatorname{NIS}\left(C_{n}^{+++}\right)=\left\{\begin{array}{c}
0 n \equiv 0(\bmod 3) \\
-1, \text { diğ } e r \text { durum }
\end{array}\right.
$$

İspat. $C_{n}^{+++}$transformasyon grafı,$V(G) \cup E(G)$ tepeler ve ayrıtlar kümesine sahiptir. X, $C_{n}^{+++}$grafının kapalı komşuluğunun subversion stratejisi olsun. İspat tepe sayısına bağlı olarak iki durumda incelenmelidir. 
Durum 1. $\mathrm{n} \equiv 0(\bmod 3)$ için $C_{n}^{+++}$grafindan $|X|=$ r tepe seçildiğinde grafta geriye kalan izole tepe sayısı en fazla $r$ tanedir. Bu durumda

$$
\operatorname{NIS}\left(C_{n}^{+++}\right)=i\left(C_{n}^{+++} / \mathrm{X}\right)-|x| \leq r-r=0
$$

olur.

$X=\frac{n}{3}$ seçildiğinde $r=\frac{n}{3}$ olur. Buradan;

$$
\operatorname{NIS}\left(C_{n}^{+++}\right)=\frac{n}{3}-\frac{n}{3}=0
$$

Durum 2. $n \not \equiv 0(\bmod 3)$ için $C_{n}^{+++}$grafndan atılan subversion strateji eleman sayısı $r$ olsun, izole kalan tepe sayısı en fazla $r-1 \mathrm{dir}$.

$$
\operatorname{NIS}\left(C_{n}^{+++}\right)=i\left(C_{n}^{+++} / X\right)-|X| \leq r-1-r=-1
$$

olur.

Bu durumda $|X|=\lfloor n / 3\rfloor+1$ seçilirse $i\left(C_{n}^{+++}\right)=\lfloor n / 3\rfloor$ olur ve buradan

$$
\operatorname{NIS}\left(C_{n}^{+++}\right)=-1
$$

elde edilir.

Teorem4.4. $P_{n}^{-++}, P_{n}$ yol grafının transformasyon grafı olmak üzere

$$
\operatorname{NIS}\left(P_{n}^{-++}\right)=\left\{\begin{array}{c}
0, n \equiv 2(\bmod 4) \\
-1, \text { diğer durum }
\end{array}\right.
$$

İspat.$P_{n}^{-++}$grafının teoremdeki şarta göre iki durumda incelenmeli

Durum 1. $n \equiv 2(\bmod 4)$ için $X$ kümesinin kapalı komşuluğu $P_{n}^{-++}$grafından atıldğında grafta geriye kalan izole tepe sayısı en fazla $r$ tanedir. Yani $i\left(P_{n}^{-++} / X\right) \leq r$.

Bu durumda $i\left(P_{n}^{-++} / X\right) \leq r-r=0$ dir.Yani

$$
N I S\left(P_{n}^{-++}\right) \leq 0
$$

Kolayca görülebilir ki $|X|=\frac{n+2}{4}$ seçildiğinde ;

$$
\begin{aligned}
& i\left(P_{n}^{-++} / X\right)=\frac{n+2}{4} \text { olup } \\
& \quad N I S\left(P_{n}^{-++}\right)=\frac{n+2}{4}-\frac{n+2}{4}=0
\end{aligned}
$$

elde edilir.

Durum 2. $n=1,3,4$ için $P_{n}^{-++}$transformasyon grafı için $|X|=r$ tepe seçilirse graftan geriye kalan izole tepe sayısı $r-1$ tanedir.

$\mathrm{Bu}$ durumda $i\left(P_{n}^{-++} / X\right) \leq r-1-r=-1$ dir. Ardından $|X|=\left\lfloor\frac{n+1}{4}\right\rfloor+1$ seçilirse $i\left(P_{n}^{-++}\right)=\left\lfloor\frac{n+1}{4}\right\rfloor$ olup

$$
\operatorname{NIS}\left(P_{n}^{-++}\right)=-1
$$

elde edilir.

Teorem 4.5. $K_{1, n}^{-++}, n+1$ tepeli $K_{1, n}$ yıldız grafinın transformasyon grafi olmak üzere

$$
\operatorname{NIS}\left(K_{1, n}^{-++}\right)=1-n
$$

İspat. $K_{1, n}^{-++}$yıldız grafin transformasyon grafı olmak üzere; $\mathrm{X}, K_{1, n}^{-++}$grafının kapalı komşuluğunun subversion stratejisi ve $|X|=\mathrm{r}$ olsun.

i. $\quad \mathrm{r}<\mathrm{n}$ olduğu durumlarda $i\left(K_{1, n}^{-++}\right)=0$ olacağından dolayı böyle bir seçim tanım gereği yapılamaz.

ii. $\mathrm{r} \geq \mathrm{n}$ olduğunuda $i\left(K_{1, n}^{-++}\right)=1$ olur. Buradan

$$
i\left(K_{1, n}^{-++} / X\right)-|X| \leq 1-r
$$

elde edilir. $\mathrm{f}(\mathrm{r})=1-r$ fonksiyonu azalan fonksiyon olup maksimum değerini $r=n$ değerinde alır. Buradan

$$
\operatorname{NIS}\left(K_{1, n}^{-++}\right) \leq 1-n
$$

olur.

Burada $|\mathrm{X}|=\mathrm{n}$ olacak şekilde öyle bir $\mathrm{X}$ kümesi vardır buradan $i\left(K_{1, n}^{-++}\right)=1$ olup

$$
\operatorname{NIS}\left(K_{1, n}^{-++}\right)=1-n
$$

elde edilir.

Teorem 4.6. $C_{n}^{-++}, C_{n}$ çevre grafının transformasyon grafı olmak üzere;

$$
\operatorname{NIS}\left(C_{n}^{-++}\right)=\left\{\begin{array}{c}
0, n \equiv 0(\bmod 3) \\
-1, \text { diğer durum }
\end{array}\right.
$$

İspat . Teoremin ispatı Teorem 4.3 'e benzer şekildedir.

\section{Tartıșma ve Sonuc}

Günümüzde bilgi teknolojilerindeki hızlı gelişmenin ve rekabetin etkileşimi sonucunda bir ağın güvenirliği, ne kadar sağlam kaldığı önemlidir. Komşuluk zedelenebilirlik parametreleri bir ağdaki birimlerin diğer birimleri ile arasındaki komşuluk ilişkisine göre zedelenebilirlik değeri bulur. Komşu izole saçılım sayısı büyük ise graflar için kolay zedelenebilir graflardır, küçük ise kolay zedelenmeyen graflardır. $\mathrm{Bu}$ çalışma sonucunda komşu izole saçılım sayısı yardımıyla $K_{1, n}^{-++}$transformasyon grafının kolay zedelenmediği, $K_{1, n}^{+++}$oldukça kolay zedelendiği görülmektedir. $P_{n}^{+++}, C_{n}^{-++}, P_{n}^{+++}$transformasyon graflarının ise $K_{1, n}^{-++}$ylldiz transformasyon grafindan daha zedelenebilir oldukları, $K_{1, n}^{+++}$grafına göre ise daha az zedelenebilir oldukları elde edilmiştir. 


\section{Kaynakça}

[1] Aslan, E., 2019. Average Binding Number, Scienceasia, 45, 85-91

[2] Aslan, E., 2015. Neighbour Isolated Scattering Number of Graphs. Scienceasia, 41, 423-431.

[3] Aytaç, A., Turacı, T., 2011.Vertex Vulnerability parameter of Gear Graphs, International Journal of Foundations of Computer Science, 22(5), 1187-1195.

[4] Aytaç, A., Turacı, T., 2015. Vulnerability Measures of Transformation Graph Gxy+, International Journal of Foundations of Computer Science 26(6), 667-675.

[5] Aytaç, V., 2012. Average Lower Domination Number in Graphs, Comptes Rendus l Academie Bulgare des Sciences, 65 (12), 1665-1674.

[6] Aytaç, V., 2009. Computing the Tenacity of Some Graphs, Selcuk Journal Apllied Sciences, 10,107120.

[7] Bacak-Turan, G., Kirlangic, A., 2011. Neighbor Rupture Degree and the Relations Between
Other Parameters, Ars Combinatoria, 102, 333352.

[8] Bacak-Turan, G., Kirlangic, A., 2011. Neighbor Integrity of Transformation Graphs, International Journal of Computer Sciences, 24 (3), 303-317.

[9] Cozzens, M.B., Wu, S.Y., 1996. Vertex-NeighborIntegrity of Trees, Ars Combinatoria 43, 169-180

[10] Gunther, G., 1985. Neighbor Connectivity in Regular Graph, Discrete Applied Mathematics, 11, 233-243.

[11] Harary F., 1994. Graph Theory, Addison-Wesley, NY.

[12] $\mathrm{Xu}, \mathrm{L} ., \mathrm{Wu}, \mathrm{B} .2008$. The transformation graph $\mathrm{G}^{\mathrm{xyz}}$ when $\mathrm{xyz}=-++$, Discrete Mathematics 308, 5144-5148 s.

[13] Turacl, T., Ökten, M., 2015. Vulnerability of Mycielski Graphs via Residual Closeness, Ars Combinatoria, 118, 419-427.

[14] Wei, Z.T., Mai, A., Zhai, M. 2011. Vertex-Neighbor Scattering Number of Graphs, Ars Combinatoria, 102, 417-26. 